\title{
EMG: Myths and Facts
}

\author{
Joseph Feinberg, MD, MS
}

Published online: 13 February 2006

(C) Hospital for Special Surgery 2006

Electrodiagnostic testing, more commonly known as the EMG test, to many is a "black box." It is a commonly ordered test that can provide very definitive information that is often not well understood. A large amount of information is usually provided, yet most clinicians rely primarily on the concluding statements, which may or may not be well substantiated. Added insight into this test demands understanding some basic facts about the "EMG" test and dispelling some of the myths.

\section{The timing for ordering an EMG study}

One of the most common myths about EMG tests is that one must wait 2-3 weeks following a nerve injury before reliable information can be obtained. It is true that the degree of muscle denervation that occurs after nerve injury can not be determined until Wallerian degeneration is complete and this can take as short as 1 week or as long as 4 weeks [1]. This is a length-dependent process so that the longer the length of the injured axon, the longer Wallerian degeneration will take. However, when there is significant axonal injury or even a severe neuropraxia (conduction block), a decreased rate of motor unit recruitment will instantaneously become abnormal and a skilled electromyographer can often determine this. These early findings can help to localize the site of injury and to some extent the degree of injury.

Nerve conduction studies across an injured segment of nerve will also be abnormal immediately. The limitation here is that one can not differentiate a conduction block where the nerve/axon remains intact from axonal degeneration or from nerve/axon transection. However, electrodiagnostic studies can never truly tell whether there is axon transection or not.

\section{Determining neurological recovery}

EMG testing can be used to demonstrate neurological recovery. Peripheral neurological recovery occurs in two forms: true axonal regeneration and the reinnervation of denervated muscle fibers by terminal collateral sprouting.

J. Feinberg, MD, MS ( $\square)$

Physiatry Department at the Hospital for Special Surgery,

Hospital for Special Surgery,

535 East 70th Street, New York, NY 10021, USA

e-mail: feinbergj@hss.edu

True axonal regeneration occurs at the site where the nerve has been injured and where axonal degeneration begins [2]. For axonal regeneration to occur, there must be an intact anterior horn cell also known as the motor neuron cell body and an intact channel for regeneration, the endoneurial tube. When axonal injury occurs, the axon distal to the site of injury undergoes Wallerian degeneration, whereas the proximal axonal component usually remains intact. The proximal axon forms a bud that begins to regenerate distally through the endoneurial tube toward the denervated muscle. This process occurs at a rate of 3-4 mm/day so axonal regeneration is length-dependent $[3,4]$.

Neurological recovery is also dependent on healthy viable muscle tissue. This is necessary for the release of nerve growth factors from denervated muscle. These factors act as a catalyst to stimulate the axon to regenerate. If the denervated muscle becomes fibrotic, these factors may no longer be released. Muscle tissue must also remain viable and electrically active if a regenerating axon is going establish a connection with a functional neuromuscular junction. Chronically denervated muscle will eventually become fibrotic and electrically inactive. This usually happens somewhere between 18 and 24 months.

The second form of peripheral neurological recovery is terminal collateral sprouting. This is the reinnervation of denervated muscle fibers by small nerve sprouts that arise from nearby uninjured and intact axons [5]. The most terminal portion of these axons generates sprouts that grow until they innervate the denervated muscle fibers. This process occurs much earlier than true axonal regeneration because the distances are much shorter. A motor unit is defined as all the muscle fibers innervated by one anterior horn cell and its axon. There is a limit to how much terminal collateral sprouting a single motor unit can undergo. It has been estimated that a motor unit can reinnervate denervated muscle tissue until it has innervated five times the amount of muscle tissue it had originally innervated $[6,7]$.

EMG testing can identify and distinguish between these two processes. Electrophysiologically, a normal motor unit has a di- or triphasic configuration. Polyphasic potentials are abnormal electrical configurations of a motor unit and can be identified following axonal injury. Two types of polyphasic potentials can form following axonal degeneration: (1) nascent potentials (Fig. 1a) and (2) motor units 

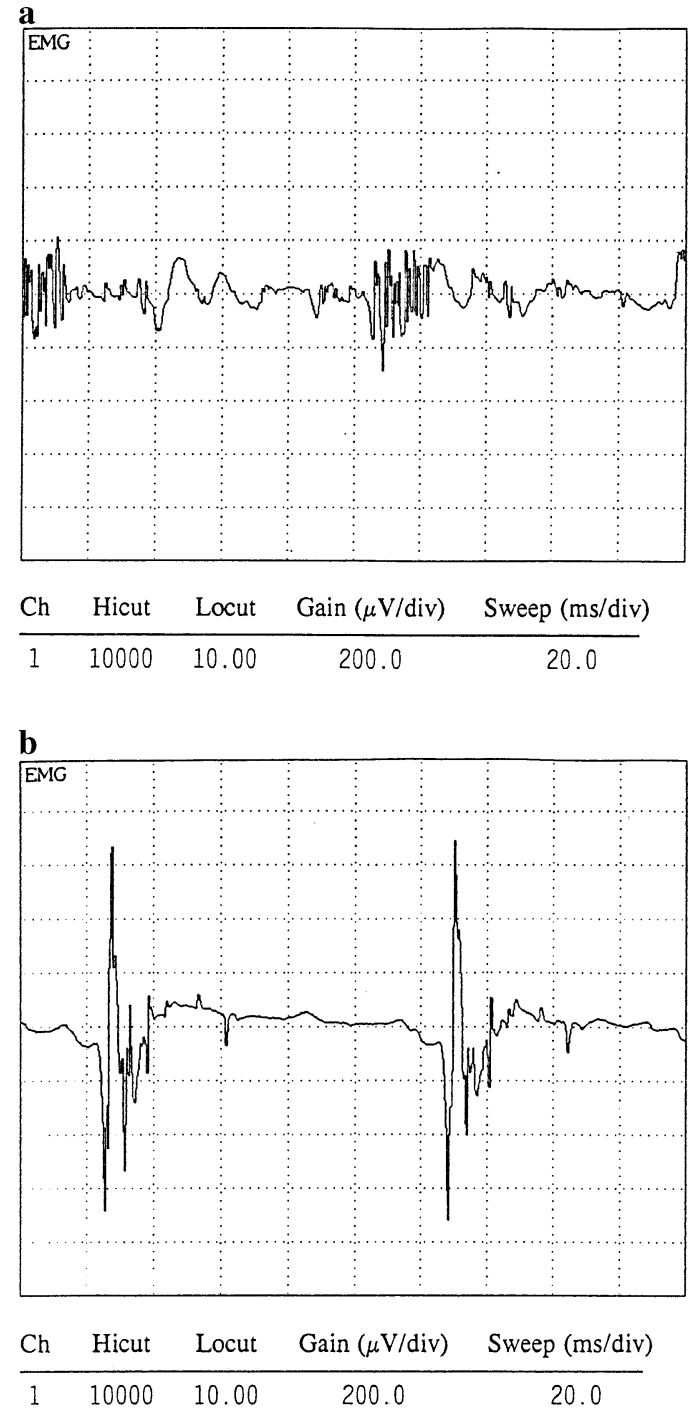

Fig. 1. a A nascent potential from axonal regeneration. b A long duration polyphasic potential from terminal collateral sprouting

formed from terminal collateral sprouting (Fig. 1b). True axonal regeneration leads to the formation of nascent potentials, which are usually low in amplitude, polyphasic in configuration, and can have a short or normal and sometimes even long duration. Terminal collateral sprouting always leads to the formation of long-duration polyphasics. Although both are polyphasic, their configuration is distinctly different and it is important to remember that only the nascent potentials represent true axonal regeneration. This important distinction is often overlooked in many EMG reports. Understanding the difference between the two and performing a careful critical analysis should usually enable an electromyographer to determine what type of polyphasic is being identified and what it indicates.

\section{Prognosticating outcome}

Frequently, after a neurological injury an individual will show signs of improved strength and function. Although this could represent a form of neurological recovery, other factors can lead to similar results. Adaptive biomechanical changes, functional compensation, and muscle substitution patterns can all lead to improved strength and function. These may give the appearance of neurological recovery when in fact there may be none. EMG needle testing can definitively answer this question. If there are no active motor units in a given muscle, then neurological recovery has not yet occurred.

Conversely, an individual may show no improvement in strength several months after a nerve injury. The clinical impression is that there has been no recovery. These findings would increase the suspicion of a nerve transection. The decision of surgical intervention must then be entertained. When axonal regeneration occurs, the initial immature nascent potentials are small and may be incapable of generating a significant or a detectable force. EMG testing at this stage may identify these early immature motor units. This not only indicates neurological recovery when clinically there appeared to be none, but also prognosticates that additional functional recovery should occur in time. This also establishes the fact that there is axonal continuity.

\section{Differentiating acute vs chronic injuries}

The question of whether neurological deficits are the result of an acute or chronic injury often arises. Although nerve conduction studies are very sensitive for localization, they are not effective for determining chronicity. It is the needle exam or EMG component that can help define whether the deficits are the result of acute and/or chronic injury.

The earliest manifestation of axonal denervation is the presence of positive sharp waves and fibrillation potentials. As noted earlier, it takes between 1 and 4 weeks after a nerve injury before they can be identified. These early denervation potentials will be large in size on the EMG screen. Months later, the fibrillation potentials and positive sharp waves become smaller. Initially, fibrillation potentials may be as larger as $1 \mathrm{mV}$ [1] in size, whereas the mean fibrillation amplitude within the first 2 months is $600 \mu \mathrm{V}$ [8]. At 6 months the mean amplitude drops by $50 \%$ to $300 \mu \mathrm{V}$, and at 1 year will be $100 \mu \mathrm{V}$ or smaller [9]. As a rule, larger amplitudes suggest nerve injury within the past few months, whereas the smaller amplitudes suggest that injury occurred at least 6 months or a year ago.

Motor unit configuration can also be used to determine whether a nerve injury is acute or chronic. Polyphasic motor units usually indicate chronicity except in myopathic disorders. As discussed earlier, it usually takes several months before nascent potentials appear. Based on the length of the distal component of the injured nerve necessary for axonal regeneration, one can often roughly estimate the amount of time regeneration will take. For example, injury to the lower trunk of the brachial plexus resulting in denervation of the hand intrinsics may take a year and a half before one would expect to see a nascent potential. An injury to the axillary nerve leading to 
denervation of the deltoid may take less than 6 months before nascent potentials could be identified.

The polyphasic motor units that result from terminal collateral sprouting as mentioned earlier look very different from nascent potentials and form much sooner than nascent potentials. Generally, the time frame for the sprouts to reinnervate denervated muscle tissue should roughly be the same regardless of the length of the injured nerve. This is because the sprouts form from the most distal portion of uninjured axons and must only grow the short distance necessary to connect to the neuromuscular junction of that same muscle. The distance is always short and so these polyphasics may be seen as early as 6-8 weeks. As the myelin of terminal collateral sprouts mature, these long duration polyphasics shorten and the main motor unit becomes bigger in time as they begin to summate. Eventually, the motor unit may grow extremely large and if enough muscle tissue is denervated and reinnervated by sprouts from the same motor unit, it may grow large enough to become a "giant motor unit." The giant motor units are greater than $8 \mathrm{mV}$ and usually take a long time to reach their final size, often taking several years. They are classically seen in postpolio patients, in slowly progressive inherited conditions, and in some very chronic acquired axonal neuropathies.

\section{Precise localization of the nerve injury}

Localization of a peripheral nerve injury is one of the most common reasons a study is being performed. Both the nerve conduction studies and EMG part of the exam help to localize the site but the nerve conduction studies are more precise. This is because one can usually stimulate the nerve at multiple sites. This is known as segmental stimulation [10-12]. At the point of injury there will be either focal slowing or a sudden change in the waveform configuration. Nerve conduction studies can often localize injury in early/ mild neuropraxias.

Localization via the needle exam is usually based on identifying muscles with the denervation potentials (fibrillation potentials and positive sharp waves). By identifying muscles with and without the denervation potentials, one can usually map out and approximately estimate where the injury is. The limitation here is that most neuropraxias do not yield denervation potentials. However, as mentioned previously, a significant change in motor unit recruitment can also be detected on the needle exam. Severe neuropraxias can lead to marked decreases in motor unit recruitment that can also be used to map out and estimate the level of nerve injury.

\section{Judging the quality of an EMG report}

A good-quality EMG report should always include the actual waveforms of sensory, motor, and $\mathrm{F}$ wave tests. This is in addition to the numerical data that are usually included. The exclusion of this prevents a more complete interpretation of the report.

Another important indicator of a good-quality study is the inclusion of temperature data. Temperature can have an im- portant effect on the nerve conduction studies. Cold temperatures will slow down nerve conduction velocities and could lead to falsely prolonged distal latencies or slow conduction velocities. Skin temperature is monitored with a temperature probe that connects to the EMG machine. Temperature is usually measured alongside the recording electrodes. It is usually recommended that temperature be at least $30^{\circ} \mathrm{C}$ in the lower limbs and $32^{\circ} \mathrm{C}$ in the upper limbs. A heat lamp or warm compresses are used to warm the limb, but there are occasions where the desired temperature can not be obtained and in these cases one can use a conversion factor to account for the cold temperature. Whether or not the ideal temperature can be obtained, an EMG report without a temperature recorded offers incomplete and possibly inaccurate information.

Electrodiagnostic studies should always be considered an extension of the physical exam. The final diagnosis and subsequent clinical decisions made should reflect that. Studies of the contralateral asymptomatic side may sometimes be indicated for comparison when there are abnormal findings.

\section{Conclusion}

Electrodiagnostic studies provide important diagnostic information often essential for optimal patient management. A clinician requesting these studies will benefit from knowing the proper timeframe for ordering them, what information can be obtained and is specifically needed, and whether a study is of good quality.

\section{References}

1. Dumitru D, Zwarts MJ (2005) Needle electromyography. In: Dumitru D, Amato AA, Zwarts MJ (eds) Electrodiagnostic medicine, Hanley and Belfus, Philadelphia, pp 257-292

2. Herbison GJ (1984) Neuropathic Needle Examination. AAEM Course A: Fundamentals of EMG, American Association of Electrodiagnostic Medicine, Rochester, MN, pp 21-25

3. Berenberg RA, Forman DS, Wood DK et al. (1977) Recovery of peripheral nerve function after axonotomy. Effect of triiodothyronine. Exp Neurol 57:349-363

4. Forman BS, Berenberg RA (1978) Regeneration of motor axons in the rat sciatic nerve studied by labeling with axonally radioactively protein. Brain Res 156:387-436

5. Wohlfart G (1958) Collateral regeneration in partially denervated muscles. Neurology 8:175-180

6. Brown MC, Ironton R (1978) Sprouting and regression of neuromuscular synapses in partial denervated mammalian muscles. J Physiol 278:325-348

7. Thompson W, Jansen JKS (1977) The extent of sprouting of remaining motor units in partially denervated immature and adult soleus muscle. Neuroscience 2:523-535

8. Kraft GH (1990) Fibrillation potential amplitude and muscle atrophy following peripheral nerve injury. Muscle Nerve 13:814-821

9. Buchthal F (1982) Fibrillations: clinical electrophysiology. In: Culp WJ, Ochoa J, (eds) Abnormal nerves and muscle generators, Oxford University Press, New York, pp 632-662

10. Campbell WW (2002) Electrophysiological approaches to the diagnosis and assessment of ulnar neuropathy. 2002 AAEM Plenary Session Toronto, Ontario, American Association of Electrodiagnostic Medicine, pp. 13-22

11. Dumitru D, Zwarts MJ (2002) Focal peripheral neuropathies. In: Dumitru D, Amato AA, Zwarts MJ, (eds) Electrodiagnostic medicine, Hanley and Belfus, Philadelphia, pp 1043-1126

12. Kimura J (1979) The carpal tunnel syndrome. Brain 102:619-635 